\title{
Development of oil-in-water microemulsion encapsulating Vitamin E for thermal and hydrolysis degradation study
}

\author{
Khairun Nisa Abdul Rahman a, Vicit Rizal Eh Suk ${ }^{a}$, Khalisanni Khalid ${ }^{b}$, Nurhazirah Mohd Ihsan ${ }^{\mathrm{c}}$, \\ Zainurin Md Dom ${ }^{\mathrm{c}}$, Misni Misran ${ }^{\mathrm{a}, *}$
}

a Colloid Laboratory, Department of Chemistry, Faculty of Science, University of Malaya, 50603 Kuala Lumpur, Malaysia

b Agri-Nanotechnology Program, Biotechnology, and Nanotechnology Research Centre, Malaysian Agriculture Research and Development Institute (MARDI), MARDI Headquarters, Persiaran MARDI-UPM, 43400 Serdang, Selangor, Malaysia

c Sime Darby Research Sdn Bhd (Bioganic), $9^{\text {th }}$ Mile, Klang Banting Road, Telok Panglima Garang Industrial Estate, 42500 Kuala Langat, Selangor Darul Ehsan, Malaysia

* Corresponding author: misni@um.edu.my

\section{Article history}

Received 28 March 2019

Revised 9 October 2019

Accepted 20 November 2019

Published Online 15 June 2020

Graphical Abstract

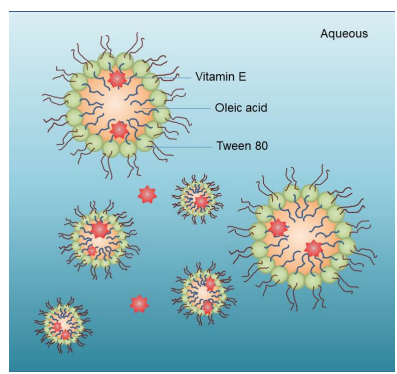

\section{Abstract}

Vitamin E is widely used for medicinal and cosmeceuticals purposes. However, it is easy to degrade by the environment. In this study, the degradation of Gold Tri.E ${ }^{\mathrm{TM}}$ was studied and determined. Gold Tri.E ${ }^{\mathrm{TM}}$ is a mixture of Vitamin $\mathrm{E}$ homologs (tocotrienol and tocopherol) extracted from palm oil (Elais Guineensis). A nanocarrier system has been optimized to encapsulate Gold Tri.E ${ }^{\mathrm{TM}}$ from degrading and increasing its stability as a bioactive compound. An oil-in-water (o/w) microemulsion was formulated and optimized as the best carrier to encapsulate Gold Tri.E ${ }^{\mathrm{TM}}$ with the mean particle size of $32.60 \pm 3.60 \mathrm{~nm}$ and $99.99 \pm 0.01 \%$ encapsulation efficiency (EE). Degradation of the Gold Tri.E ${ }^{\text {TM }}$ in o/w microemulsion was significantly reduced as compared to the bare Gold Tri. $\mathrm{E}^{\mathrm{TM}}$. This suggested that the system could protect Gold Tri.E ${ }^{\mathrm{TM}}$ from thermal and hydrolysis degradation. Thus, the ease of preparation, low-cost production, and small particle size obtained when Gold Tri.E ${ }^{\mathrm{TM}}$ encapsulated in this system give promising drug delivery system to encapsulate, protect, and increase the shelf life of Gold Tri.E ${ }^{\mathrm{TM}}$.

Keywords: Vitamin E, tocopherol, tocotrienol, degradation study, microemulsion

\section{INTRODUCTION}

Vitamin is an essential micronutrient that an organism needs in small quantities for the proper functioning of its metabolism. Vitamins can be water-soluble or lipid-soluble. Most vitamins are not single molecules, but groups of related molecules called vitamers. Vitamin E is a lipid-soluble vitamin that consists of tocopherols and tocotrienol (Sarbandi et al., 2018). The chemical structures of vitamin E homologs, which are tocotrienol and tocopherol, were depicted in Fig. 1 (a) and (b), respectively.

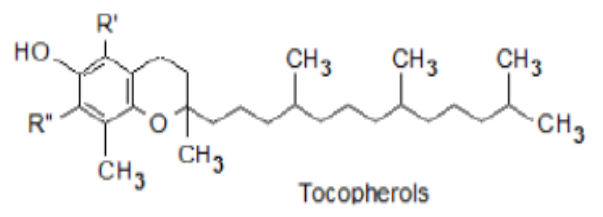

where,

$\alpha$-Tocopherol: $\mathrm{R}^{\prime}=\mathrm{R}, '=\mathrm{CH} 3$

$\beta$-Tocopherol: $\mathrm{R}^{\prime}=\mathrm{CH} 3, \mathrm{R}$ ' $=\mathrm{H}$

$\gamma$-Tocopherol: $\mathrm{R}^{\prime \prime}=\mathrm{CH} 3, \mathrm{R}^{\prime}=\mathrm{H}$

$\delta$-Tocopherol: R'=R'"=H

Fig. 1(a) Chemical structure of vitamin E homologs, which is tocopherol.

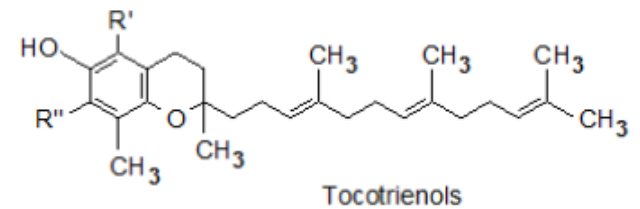

where,

$\alpha$-Tocotrienol: $\mathrm{R}^{\prime}=\mathrm{R}$ ' $=\mathrm{CH} 3$

$\beta$-Tocotrienol: $\mathrm{R} '=\mathrm{CH} 3, \mathrm{R}$ ' $=\mathrm{H}$

$\gamma$-Tocotrienol: R' $=\mathrm{CH} 3, \mathrm{R} '=\mathrm{H}$

$\delta$-Tocotrienol: $\mathrm{R}^{\prime}=\mathrm{R}$ ' $=\mathrm{H}$

Fig. 1(b) Chemical structure of Vitamin E homologs, which is tocotrienol.

Primary dietary sources of vitamin E include vegetable oils, seeds, and cereal grains (Combs \& McClung, 2017). It has a fundamental role as an antioxidant in the metabolism of all cells, which scavenges free radicals and protects membrane lipid from oxidation (Combs \& McClung, 2017; Devereux et al., 2019). However, Vitamin E is highly susceptible to degradation in the ambient environments, such as temperature, light, and humidity (Hategekimana \& Zhong, 2015).

Hence, the colloidal carrier system is one of the best alternatives that can be applied in the aim to protect and deliver 
the lipophilic bioactive component to the targeted site (Aggarwal et al., 2010; Hategekimana \& Zhong, 2015). Various nanocarriers have been explored to encapsulate vitamin $\mathrm{E}$, such as liposome (Amiri et al., 2018), nanostructured lipid (Ying \& Misran, 2017), polymeric nanoparticles (Zhang et al., 2017) and emulsion (Alayoubi et al., 2013; Yang \& McClements, 2013; Raikos, 2017). In this study, we explored the formulation of microemulsion as a protective carrier for vitamin $\mathrm{E}$ from degradation due to the simpler laboratory preparation, which giving more advantages to scale up at the industrial level and involving the low cost of production (McClements, 2012).

Gold Tri.ETM, which is a trademark product of Sime Darby Plantation Berhad, would be used as a vitamin E model. Gold Tri.ETM contains a mixture of tocotrienol and tocopherol that extracted from Elais Guineensis palm fruit (Heng et al., 2013). Gold Tri.E TM is yellow and commonly used as a dietary supplement, nutraceutical, functional food ingredient, and cosmeceutical (Muhammad et al., 2012). By incorporating the Gold Tri. $\mathrm{E}^{\mathrm{TM}}$ into oil-in-water (o/w) microemulsion, the Gold Tri.E ${ }^{\mathrm{TM}}$ would be expected to be protected from degradation. Furthermore, its small average particle size could enhance the delivery of Gold Tri. $\mathrm{E}^{\mathrm{TM}}$ to the targeted site and improve the efficacy of Gold Tri. $\mathrm{E}^{\mathrm{TM}}$.

\section{EXPERIMENTAL}

\section{Materials}

The Gold Tri.ETM 20P, 30P, 50, and 70 were generously provided by Sime Darby Food \& Beverage Marketing Sdn. Bhd. (Selangor, Malaysia). Oleic acid and Tween 80 were purchased from Fluka (Buchs, Switzerland). All samples were prepared by using deionised water with the resistivity of $18.2 \Omega \mathrm{cm}^{-1}$, collected from a Barnstead Diamond Nanopure Water Purification unit that was pre-filtered by Barnstead Diamond ${ }^{\mathrm{TM}}$ RO unit (Barnstead International, USA).

\section{Fourier transform infrared spectroscopy (FTIR)}

Functional group analysis of Gold Tri.ETM 20P, 30P, 50, and 70 was characterized by using Spectrum 400 Fourier TransformInfrared (FT-IR) Spectrometer (Perkin Elmer, USA) equipped with attenuated total reflectance (ATR) accessory (GladiATRPIKE Technologies, USA) in the Department of Chemistry, UM. Five mg of sample was placed on the clean, residue-free ATR crystal surface and scanned at $4 \mathrm{~cm}^{-1}$ within the wavenumbers of 450 to $4000 \mathrm{~cm}^{-1}$.

\section{Differential scanning calorimetry (DSC)}

Thermal behaviour of Gold Tri.ETM 20P, 30P, 50, and 70 was analyzed by using a Shimadzu DSC-60 Differential Scanning Calorimeter (Shimadzu, Kyoto, Japan). The DSC has been precalibrated with indium (Aboulfazli et al., 2014). About $10 \mathrm{mg}$ of the sample was carefully transferred into Tzero Aluminum Hermetic DSC pans and placed in the DSC cell by using the empty pan as a standard pan. Both sample pans were heated to $180{ }^{\circ} \mathrm{C}$ with the scan rate of $10{ }^{\circ} \mathrm{C} \mathrm{min}-1$ in the same single furnace. The heating run was performed with a continuous flow of nitrogen at $50.0 \mathrm{ml} \mathrm{min}$.

\section{Preparation of oil-in-water (o/w) microemulsion}

Five $\mathrm{g}$ of oleic acid was mixed with $10 \mathrm{~g}$ of deionized water with the presence of $2,5,10,20$, or $25 \% \mathrm{w} / \mathrm{w}$ of Tween 80 . The mixtures were undergone mild stirring at ambient temperature $\left(24.8 \pm 0.1{ }^{\circ} \mathrm{C}\right)$. The $\mathrm{o} / \mathrm{w}$ microemulsion obtained was left for about 30 minutes prior to analysis. The formulation of the $\mathrm{o} / \mathrm{w}$ microemulsion was as depicted in Table 1. The optimum formulation was then further incorporated with $0.05 \% \mathrm{w} / \mathrm{w}$ of Gold Tri.E 30P. The o/w microemulsion was then incubated at room temperature for further analysis.

Table 1 Formulation of the o/w microemulsion.

\begin{tabular}{cccc}
\hline \multirow{2}{*}{ Formulation } & \multicolumn{3}{c}{ Microemulsion composition (\% w/w) } \\
\cline { 2 - 4 } & Tween $\mathbf{8 0}$ & Oleic Acid & Water \\
\hline M1 & 0 & 5 & 95 \\
M2 & 2 & 5 & 93 \\
M3 & 5 & 5 & 90 \\
M4 & 10 & 4 & 86 \\
M5 & 20 & 4 & 76 \\
M6 & 25 & 4 & 41 \\
\hline
\end{tabular}

Mean particle size and polydispersity index (PDI) measurement

The mean particle size and polydispersity index (PDI) of microemulsion were measured by employing the Zetasizer Nano ZS (Malvern Instruments, U.K.). A $1 \mathrm{~cm}$ path length quartz cuvette was used for the measurement of the mean particle size and PDI measurement. The sample was equilibrated for 2 minutes at $25.0 \pm 0.1{ }^{\circ} \mathrm{C}$ and the measurement was done in triplicates.

\section{Encapsulation efficiency of Gold Tri.E ${ }^{\mathrm{TM}}$}

The supernatant at the bottom of the vials was carefully collected and analyzed by a UV-Vis spectrophotometer. The amount of free Gold Tri.ETM 30P was determined from the standard calibration curve of Gold Tri.ETM $30 \mathrm{P}$ at $295 \mathrm{~nm}$ with the coefficient of determination, $R^{2}$, of 0.9940 . Standard calibration curve of Gold Tri.ETM $30 \mathrm{P}$ is available upon request. Encapsulation efficiency (\%EE) for each formulation was determined by using equation (1).

$$
\% E E=\left(W_{T}-W_{F}\right) / W_{T} \times 100 \%
$$

where $W_{T}$ is the weight of Gold Tri. $E^{\mathrm{TM}}$ added to the formulation and $W_{F}$ is the weight of the free Gold Tri. $\mathrm{E}^{\mathrm{TM}}$.

\section{Degradation study by UV-Vis spectroscopic method}

Degradation of Gold Tri.E ${ }^{\mathrm{TM}} 30 \mathrm{P}$ was analysed by using Cary $50 \mathrm{UV}$-Vis Spectrophotometer (Agilent Technologies, USA). The o/w microemulsion, Gold Tri.ETM 30P, and o/w microemulsion encapsulating Gold Tri.ETM 30P were carefully transferred into glass vials and incubated at 8.0, 25.0, or $45.0 \pm 0.1{ }^{\circ} \mathrm{C}$ in the absence of light for 1 hour. The amount of Gold Tri.ETM 30P presented after incubation was quantified from the standard calibration curve at $295 \mathrm{~nm}$.

\section{RESULTS AND DISCUSSION}

\section{Characterization of Gold Tri.E ${ }^{\mathrm{TM}}$}

Fig. 2 shows the FTIR spectra of Gold Tri.ETM 20P, 30P, 50, and $70 \%$ containing the mixture of vitamin $\mathrm{E}$ homologs. The broad peak at 3331 to $3433 \mathrm{~cm}^{-1}$ was attributed to the $\mathrm{OH}$ stretching bend. The peaks within 2925 to $2854 \mathrm{~cm}^{-1}$, showed the presence of asymmetric and symmetric stretching vibrations of the $-\mathrm{CH}_{2}$ and $-\mathrm{CH}_{3}$ (Silva et al., 2009). The characteristic peak at $1450 \mathrm{~cm}^{-1}$ showed the presence of phenyl skeletal, while methyl asymmetric bending at $1440 \mathrm{~cm}^{-1}$ and symmetric bending at $1377 \mathrm{~cm}^{-1}$. The phenyl skeletal stretch and methyl asymmetric bending were overlapped with each other with respect to the presence of shoulder shown in the spectra. Peaks at 1240 to $1220 \mathrm{~cm}^{-1}$ were corresponded to the $-\mathrm{CH}_{2}$, while peak 
$1086 \mathrm{~cm}^{-1}$ was for the plane bending of phenyl and peak $928 \mathrm{~cm}^{-}$ ${ }^{1}$ was represented the trans $=\mathrm{CH}_{2}$ stretching (Man et al., 2005). There was also a strong peak shown at $1087 \mathrm{~cm}^{-1}$, indicating the presence of C-O stretch. An overtone peak at 1850 to $2340 \mathrm{~cm}^{-}$ ${ }^{1}$ was a signal from the combination of bands and assigned as the ring substitution pattern of the phenyl ring. The peaks from the vibration motions, as showed in Fig. 2, reconfirmed the structure of Tri.ETM that contained Vitamin E homologs, which are tocopherol and tocotrienol.

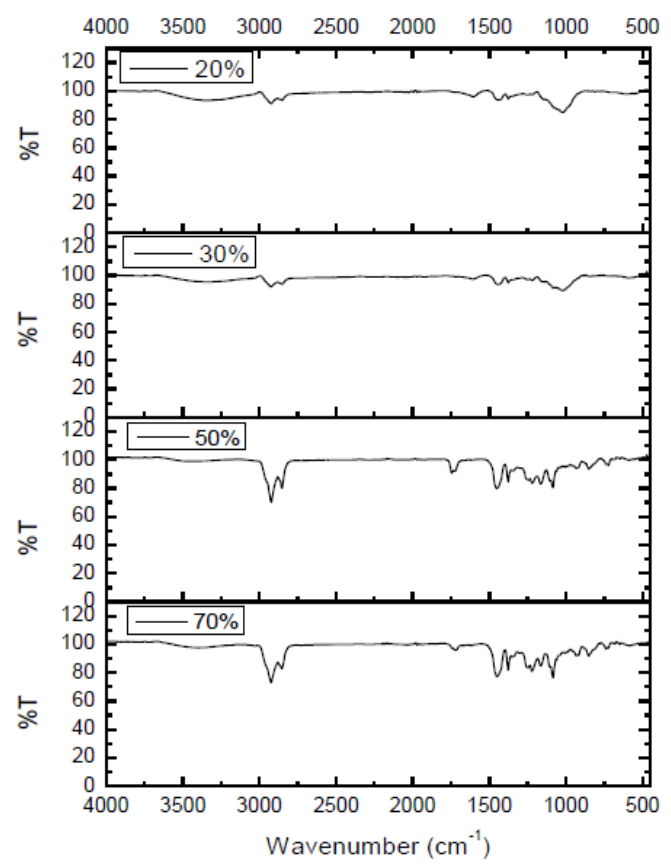

Fig. 2 FTIR spectra of Gold Tri. $E^{\mathrm{TM}} 20 \mathrm{P}, 30 \mathrm{P}, 50$, and 70 at room temperature.

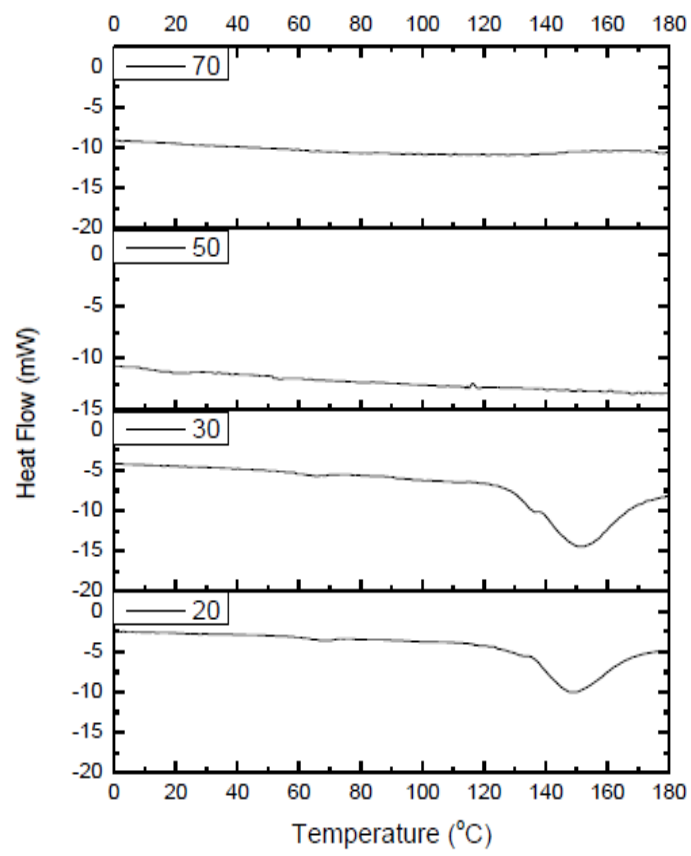

Fig. 3 Thermal behaviour of Gold Tri.E ${ }^{\mathrm{TM}} 20 \mathrm{P}, 30 \mathrm{P}, 50$ and 70 at $10^{\circ} \mathrm{C} \mathrm{min}^{-1}$.

\section{Thermal behaviour of Gold Tri. $\mathrm{E}^{\mathrm{TM}}$}

Fig. 3 shows the thermal behaviour of Gold Tri.ETM 20P, 30P, 50 and 70. At room temperature, Gold Tri.ETM $20 \mathrm{P}$ and $30 \mathrm{P}$ were solid, while Gold Tri.ETM 50 and 70 were in liquid form.
Even at low temperatures, Gold Tri.ETM 50 and 70 were in liquid form, resulting in the absence of melting point, $T_{m}$, within the tested temperatures. Gold Tri.ETM $20 \mathrm{P}$ and 30P displayed thermal stability with melting points at 148 and $150{ }^{\circ} \mathrm{C}$, respectively. $T_{m}, T_{p}$, and $\Delta T_{1 / 2}$ were reported in Table 2 . Gold Tri.ETM 30P was selected to be encapsulated in the $\mathrm{o} / \mathrm{w}$ microemulsion based on their highest $T_{m}$ as it will stable at a higher temperature (Hategekimana \& Zhong, 2015).

Table 2 Thermal behaviour of Tri. $\mathrm{E}^{\mathrm{TM}}$ at a scan rate of $10^{\circ} \mathrm{C} \mathrm{min}^{-1}$.

\begin{tabular}{cccc}
\hline Sample & $\mathbf{T}_{\mathbf{p}}\left({ }^{\circ} \mathbf{C}\right)$ & $\mathbf{T}_{\mathbf{m}}\left({ }^{\circ} \mathbf{C}\right)$ & $\begin{array}{c}\mathbf{\Delta} \mathbf{T}_{1 / 2} \\
\left({ }^{\circ} \mathbf{C}\right)\end{array}$ \\
\hline Tri.E 20 & 133 & 148 & 140 \\
Tri.E 30 & 134 & 150 & 141 \\
Tri.E 50 & NA & NA & NA \\
Tri.E 70 & NA & NA & NA \\
\hline
\end{tabular}

Particle size and Polydispersity Index (PDI) of o/w microemulsion

Fig. 4 shows the average particle size and PDI of $\mathrm{o} / \mathrm{w}$ microemulsion at $25.0 \pm 0.1{ }^{\circ} \mathrm{C}$. The incorporation of Tween 80 was found to affect both mean particle size and PDI of the formulation. The amount of Tween 80 in the formulation was optimized to produce the small average particle size of $\mathrm{o} / \mathrm{W}$ microemulsion and low PDI. Low particle size is needed to deliver deeper to the targeted site, while low PDI indicated the homogenous size of the particle (McClements, 2012). Generally, two trends were observed, the decrease in average particle size from M1, M2, to M3 (a) and the increase in average particle size from M4, M5, to M6 (b). These trends were happened due to the amount of Tween 80 in the formulation. Their particle size was depended on the Tween 80 composition, showing that the average particle size and PDI of $\mathrm{o} / \mathrm{w}$ microemulsion can be engineered through the composition variation of Tween 80 in the formulations lipid (Ying \& Misran, 2017).

The o/w microemulsion M3 has a transparent appearance and a mean particle size of $32.6 \pm 3.6 \mathrm{~nm}$. This was agreeable with the previous report on the colloidal dispersion that tended to become transparent when the particle size fell around $30 \mathrm{~nm}$ (McClements, 2012). This formulation was selected to encapsulate Gold Tri.ETM $30 \mathrm{P}$.

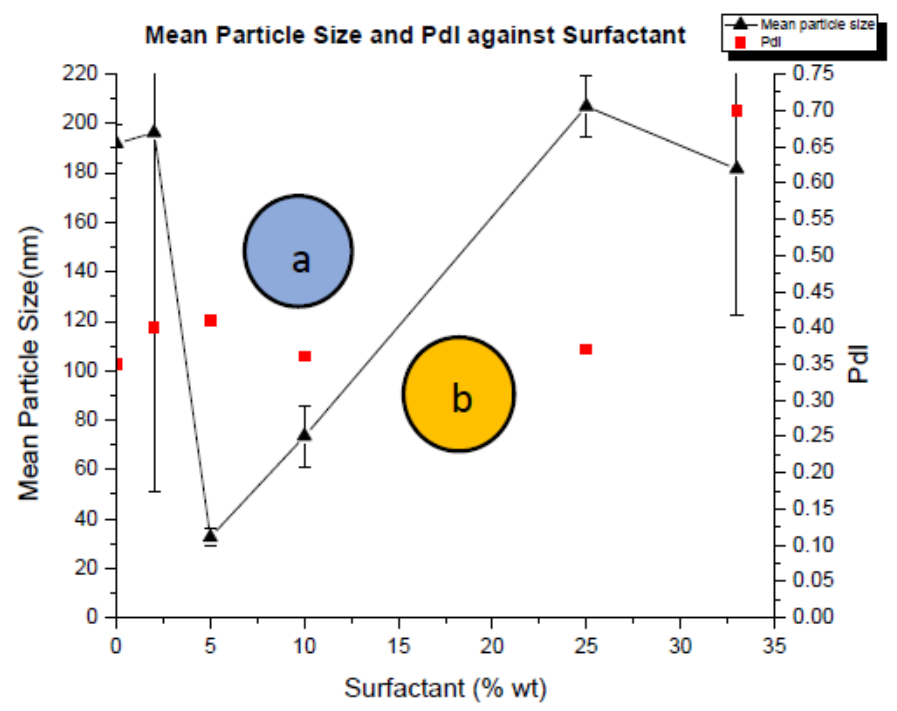

Fig. 4 Mean particle size and PDI against surfactant for the formulation of o/w microemulsion where (a) are M1, M2, and M3; (b) are M4, M5, and M6 


\section{Encapsulation efficiency (\%EE)}

Encapsulation of Gold Tri.ETM $30 \mathrm{P}$ in o/w emulsion was analyzed by measuring the free Gold Tri.ET $30 \mathrm{P}$ in the formulation and quantified from the standard calibration curve. The \%EE of Gold Tri.ETM 30P in o/w microemulsion was calculated to be $99.99 \%$, which may due to the optimized amount of surfactant in the formulation. The \%EE is improved as compared to the previous study using different formulation (Ying \& Misran, 2017). This excellent \%EE was resulted from the nature of Gold Tri.ETM 30P, which is hydrophobic. This characteristic would spontaneously force the Gold Tri.ETM 30P to be encapsulated in the core of o/w microemulsion, as depicted in the graphical abstract.

\section{Degradation study of Gold Tri.E ${ }^{\mathrm{TM}}$ in o/w microemulsion}

Vitamin E has a high tendency to experience physical instability and degradation (Hategekimana \& Zhong, 2015). Therefore, the degradation of Gold Tri.ETM 30P in the colloidal system was studied to provide the precaution steps during processing and storage.

Fig. 5 shows the variation in the amount of Gold Tri.ETM 30P as incubated in various ambient temperatures. The bare Gold Tri.ETM 30P indicated a more significant reduction in thermal degradation as compared to hydrolysis degradation. In the colloidal system, Gold Tri.ETM 30P would simultaneously undergo both thermal and hydrolysis degradations. However, M3 showed both thermal and hydrolysis stabilities against the incubated ambients. Encapsulation of Gold Tri.ETM $30 \mathrm{P}$ in o/w microemulsion showed protection against thermal and hydrolysis degradations, where there were small changes in the amount of Gold Tri.ETM $30 \mathrm{P}$ at the incubated temperature.

Thus, it emphasized that the o/w microemulsion system could be a promising system to be used as a carrier system Gold Tri.ETM $30 \mathrm{P}$ as it was able to protect the Gold Tri.ETM $30 \mathrm{P}$ from degradation.

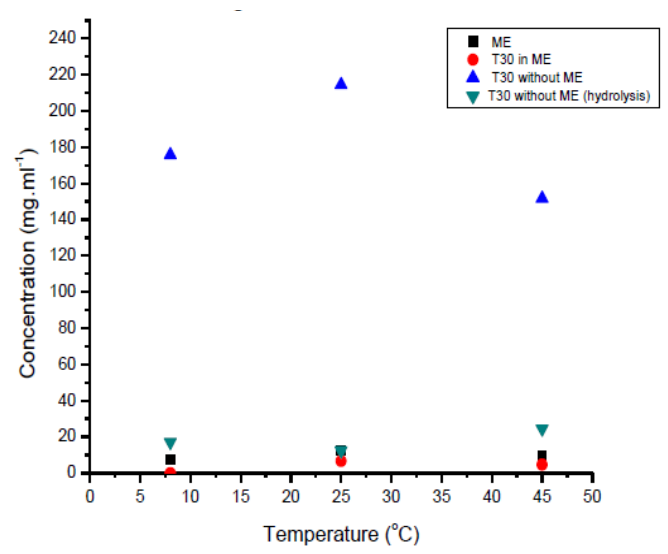

Fig. 5 The degradation of Gold Tri.E 30P in o/w microemulsion systems at different temperatures $\left(8.0,25.0\right.$, or $\left.45.0 \pm 0.1^{\circ} \mathrm{C}\right)$. T30 is Gold Tri.E $30 \mathrm{P}$ and $\mathrm{ME}$ is o/w microemulsion.

\section{CONCLUSION}

Gold Tri.ETM, which contains a mixture of Vitamin E homologs, was encapsulated in the $\mathrm{o} / \mathrm{w}$ microemulsion. The encapsulation efficiency yielded $99.99 \%$. The average particle size was $32.6 \pm 3.6 \mathrm{~nm}$, which could possibly increase the surface area and penetrate deeper into the skin. The experiments showed that o/w microemulsion could successfully protect Gold Tri.ETM $30 \mathrm{P}$ from thermal and hydrolysis degradations.

\section{ACKNOWLEDGMENT}

The authors would like to thank Sime Darby Food \& Beverage Marketing Sdn. Bhd. for kindly providing the Gold Tri.ETM. This work was financially supported by the Geran Penyelidikan Fakulti GPF065B-2018, and Fundamental Research Grant Scheme (FRGS) (FP075-2018A).

\section{REFERENCES}

Aboulfazli, F., Baba, A. S., \& Misran, M. (2014). Effect of vegetable milks on the physical and rheological properties of ice cream. Food Science and Technology Research, 20(5), 987-996.

Aggarwal, B. B., Sundaram, C., Prasad, S., \& Kannappan, R. (2010). Tocotrienols, the vitamin E of the 21 st century: Its potential against cancer and other chronic diseases. Biochemical pharmacology, 80(11), 1613-1631.

Alayoubi, A., Kanthala, S., Satyanarayanajois, S. D., Anderson, J. F., Sylvester, P. W., \& Nazzal, S. (2013). Stability and in vitro antiproliferative activity of bioactive "Vitamin E" fortified parenteral lipid emulsions. Colloids and Surfaces B: Biointerfaces, 103, 23-30.

Amiri, S., Ghanbarzadeh, B., Hamishehkar, H., Hosein, M., Babazadeh, A., \& Adun, P. (2018). Vitamin E loaded nanoliposomes: Effects of gammaoryzanol, polyethylene glycol and lauric acid on physicochemical properties. Colloid and Interface Science Communications, 26, 1-6.

Combs, G. F., \& McClung, J. P. (2017). Chapter 8 - Vitamin E. In G. F. Combs \& J. P. McClung (Eds.), The Vitamins (Fifth Edition) (pp. 207-242): Academic Press.

Devereux, G., Craig, L., Seaton, A., \& Turner, S. (2019). Maternal vitamin D and $\mathrm{E}$ intakes in pregnancy and asthma to age 15 years: A cohort study. Pediatric pulmonology, 54(1), 11-19.

Dingler, A., Blum, R., Niehus, H., Muller, R., \& Gohla, S. (1999). Solid lipid nanoparticles (SLNTM/LipopearlsTM) a pharmaceutical and cosmetic carrier for the application of vitamin $\mathrm{E}$ in dermal products. Journal of Microencapsulation, 16(6), 751-767.

Hategekimana, J., \& Zhong, F. (2015). Degradation of vitamin E in nanoemulsions during storage as affected by temperature, light and darkness. International Journal of Food Engineering, 11(2), 199-206.

Heng, E. C., Karsani, S. A., Rahman, M. A., Hamid, N. A. A., Hamid, Z., \& Ngah, W. Z. W. (2013). Supplementation with tocotrienol-rich fraction alters the plasma levels of Apolipoprotein AI precursor, Apolipoprotein E precursor, and C-reactive protein precursor from young and old individuals. European Journal of Nutrition, 52(7), 1811-1820.

Man, Y. C., Ammawath, W., \& Mirghani, M. (2005). Determining $\alpha$-tocopherol in refined bleached and deodorized palm olein by Fourier transform infrared spectroscopy. Food Chemistry, 90(1-2), 323-327.

McClements, D. J. (2012). Nanoemulsions versus microemulsions: Terminology, differences, and similarities. Soft Matter, 8(6), 1719-1729.

Muhammad, N. A., Omar, E., \& Nawawi, H. M. (2012). Reduced In-vivo Creactive protein aortic tissue expression following tocotrienol-enriched mixed fraction supplementation in cholesterol-induced atherosclerosis. Paper presented at the 2012 IEEE Colloquium on Humanities, Science and Engineering (CHUSER).

Raikos, V. (2017). Encapsulation of vitamin E in edible orange oil-in-water emulsion beverages: Influence of heating temperature on physicochemical stability during chilled storage. Food Hydrocolloids, 72, 155-162.

Sarbandi, M. S., Eshak, Z., Rahman, N. S. A., Aziz, A. T. A., Rajikin, M. H., Hashim, N., Ahmad, Z. A., \& Khan, N. A. M. N. (2018). Palm tocotrienolrich-fraction yields higher numbers of normal embryos whereas alphatocopherol produces higher preimplantation survival in murine embryos. Malaysian Journal of Fundamental and Applied Sciences, 14(3), 407-410.

Silva, S. D., Rosa, N. F., Ferreira, A. E., Boas, L. V., \& Bronze, M. R. (2009). Rapid determination of $\alpha$-tocopherol in vegetable oils by fourier transform infrared spectroscopy. Food Analytical Methods, 2(2), 120.

Yang, Y., \& McClements, D. J. (2013). Encapsulation of vitamin E in edible emulsions fabricated using a natural surfactant. Food Hydrocolloids, 30(2), 712-720.

Ying, L. Q., \& Misran, M. (2017). Rheological and physicochemical characterization of alpha-tocopherol loaded lipid nanoparticles in thermoresponsive gel for topical application. Malaysian Journal of Fundamental and Applied Sciences, 13(3).

Zhang, W., Liang, C., Liu, H., Li, Z., Chen, R., Zhou, M., Li, D., Ye, Q., Luo, C., \& Sun, J. (2017). Polymeric nanoparticles developed by vitamin Emodified aliphatic polycarbonate polymer to promote oral absorption of oleanolic acid. Asian Journal of Pharmaceutical Sciences, 12(6), 586-593. 